\title{
Correction to: Introduction to Differentiable Manifolds
}

\section{Serge Lang}

The title of this book was incorrectly captured as Introduction to Differential Manifolds. The correct title should be Introduction to Differentiable Manifolds. This has been corrected.

The updated online version of this book can be found at https://doi.org/10.1007/b97450_11 